\title{
SWIFT X-RAY AND ULTRAVIOLET MONITORING OF THE CLASSICAL NOVA V458 VUL (NOVA VUL 2007)
}

\author{
J.-U. Ness ${ }^{1,2}$, J. J. Drake ${ }^{3}$, A. P. Beardmore ${ }^{4}$, D. Boyd ${ }^{5}$, M. F. Bode ${ }^{6}$, S. Brady ${ }^{7}$, P. A. Evans ${ }^{4}$, B. T. Gaensicke ${ }^{8}$, \\ S. Kitamoto ${ }^{9}$, C. $\mathrm{Knigge}^{10}$, I. Miller ${ }^{11}$, J. P. Osborne ${ }^{4}$, K. L. Page ${ }^{4}$, P. Rodriguez-Gil ${ }^{12,13}$, G. Schwarz ${ }^{14}$, B. Staels ${ }^{15}$,

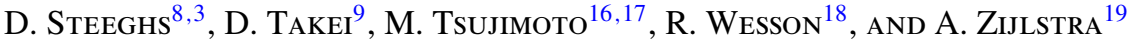 \\ ${ }^{1}$ European Space Astronomy Centre, P.O. Box 78, 28691 Villanueva de la Cañada, Madrid, Spain; juness@ sciops.esa.int \\ 2 School of Earth and Space Exploration, Arizona State University, Tempe, AZ 85287-1404, USA \\ ${ }^{3}$ Harvard-Smithsonian Center for Astrophysics, 60 Garden Street, Cambridge, MA 02138, USA \\ ${ }^{4}$ Department of Physics \& Astronomy, University of Leicester, Leicester LE1 7RH, UK \\ 5 BAA VSS, 5 Silver Lane, West Challow, Wantage, OX12 9TX, UK \\ ${ }^{6}$ Astrophysics Research Institute, Liverpool John Moores University, Twelve Quays House, Egerton Wharf, Birkenhead CH41 1LD, UK \\ 7 AAVSO, 5 Melba Drive, Hudson, NH 03051, USA \\ ${ }^{8}$ Department of Physics, University of Warwick, Coventry CV4 7AL, UK \\ ${ }^{9}$ Department of Physics, Rikkyo University, 3-34-1 Nishi-Ikebukuro, Toshima, Tokyo 171-8501, Japan \\ ${ }^{10}$ School of Physics \& Astronomy, University of Southampton, Southampton SO17 1BJ, UK \\ 11 BAA VSS, Furzehill House, Ilston, Swansea SA2 7LE, UK \\ 12 Isaac Newton Group, PO Ap. de Correos 321, 38700 Sta. Cruz de la Palma, Spain \\ 13 Instituto de Astrofísica de Canarias, Vía Láctea s/n, La Laguna, E-38205 Santa Cruz de Tenerife, Spain \\ ${ }^{14}$ Department of Geology and Astronomy, West Chester University, West Chester, PA 19383, USA \\ ${ }^{15}$ CBA Flanders, Alan Guth Observatory, Koningshofbaan 51, Hofstade, Aalst, Belgium \\ ${ }^{16}$ Department of Astronomy \& Astrophysics, Pennsylvania State University, 525 Davey Laboratory, University Park, PA 16802, USA \\ 17 Japan Aerospace Exploration Agency, Institute of Space and Astronautical Science, 3-1-1 Yoshino-dai, Sagamihara, Kanagawa 229-0015, Japan \\ ${ }^{18}$ Department of Physics \& Astronomy, University College London, Gower Street, London WC1E 6BT, UK \\ 19 Jodrell Bank Center for Astrophysics, Alan Turing Building, School of Physics and Astronomy, The University of Manchester, Oxford Street, \\ Manchester M13 9PL, UK \\ Received 2008 October 7; accepted 2009 February 12; published 2009 March 30
}

\begin{abstract}
We describe the highly variable X-ray and UV emission of V458 Vul (Nova Vul 2007), observed by Swift between 1 and 422 days after outburst. Initially bright only in the UV, V458 Vul became a variable hard X-ray source due to optically thin thermal emission at $\mathrm{k} T=0.64 \mathrm{keV}$ with an X-ray band unabsorbed luminosity of $2.3 \times 10^{34} \mathrm{erg} \mathrm{s}^{-1}$ during days 71-140. The X-ray spectrum at this time requires a low Fe abundance $\left(0.2_{-0.1}^{+0.3}\right.$ solar), consistent with a Suzaku measurement around the same time. On day 315 we find a new X-ray spectral component which can be described by a blackbody with temperature of $k T=23_{-5}^{+9} \mathrm{eV}$, while the previous hard X-ray component has declined by a factor of 3.8. The spectrum of this soft X-ray component resembles those typically seen in the class of supersoft sources (SSS) which suggests that the nova ejecta were starting to clear and/or that the white dwarf photosphere is shrinking to the point at which its thermal emission reaches into the X-ray band. We find a high degree of variability in the soft component with a flare rising by an order of magnitude in count rate in 0.2 days. In the following observations on days 342.4-383.6, the soft component was not seen, only to emerge again on day 397. The hard component continued to evolve, and we found an anticorrelation between the hard X-ray emission and the UV emission, yielding a Spearman rank probability of $97 \%$. After day 397, the hard component was still present, was variable, and continued to fade at an extremely slow rate but could not be analyzed owing to pile-up contamination from the bright SSS component.
\end{abstract}

Key words: novae, cataclysmic variables - stars: individual (V458 Vul)

\section{INTRODUCTION}

Classical novae (CNe) are caused by nuclear explosions in cataclysmic variables. A history of accretion of hydrogenrich material from a main-sequence star onto the white dwarf (WD) primary can lead to sufficient pressure in the surface envelope for thermonuclear runaway to occur. Enough radiation is produced to launch a radiatively driven wind that ejects the outer envelope and forms an optically thick shell around the WD within which the high-energy radiation that is produced by nuclear burning is initially trapped. The radiative output of the nova then occurs primarily in the optical, but as the mass ejection rate decreases, the radius of approximately unit opacity shrinks, and successively hotter layers become visible (see, e.g., Gallagher \& Starrfield 1978). If nuclear burning continues long enough, the peak of the spectral energy distribution (SED) eventually shifts into the X-ray regime, and at that time the nova emits an X-ray spectrum that resembles those typically observed in the class of supersoft X-ray sources (SSS; Kahabka $\&$ van den Heuvel 1997). For a review of nova evolution see, e.g., Starrfield et al. (2008).

Early X-ray emission has been detected in several cases that was much harder than typical SSS spectra (e.g., Lloyd et al. 1992; Krautter et al. 1996; Mukai \& Ishida 2001; Orio et al. 2001). The origin of this emission is not entirely clear because it has never been observed with sufficient signal-to-noise ratio $(\mathrm{S} / \mathrm{N})$ and spectral resolution to constrain theoretical models. The observed spectra are generally a good match to optically thin plasma models (e.g., Krautter et al. 1996; Balman et al. 1998; Orio et al. 2001), which suggests that the plasma is collisional. Mukai \& Ishida (2001) argued that shocks within the nova ejecta were responsible, and this is consistent with earlier work by Lloyd et al. (1992) and O'Brien et al. (1994). In some cases, hard emission line spectra have also been observed after the nova had turned off (e.g., V382 Vel: Ness et al. 2005). This has been attributed to nebular emission that originates 
from the surrounding medium that had been heated by radiation from the nova. However, some of the emission lines that have been observed arise at energies too high to be explained by photoexcitation. It is possible that these lines are part of the continuing hard component, which would imply that the cooling timescales of the hard component are extremely long, up to years.

A different situation is the case of symbiotic novae such as RS Oph, where the secondary is a giant with a dense stellar wind. The ejecta run into the stellar wind, dissipating some kinetic energy, producing X-ray radiation. The luminosity of the emission from these shock systems is much higher than that observed in classical novae, and well-exposed X-ray spectra with high spectral resolution have been obtained (Ness et al. 2009). In classical novae, the secondary is a main-sequence star where the wind is not dense enough for this scenario (O'Brien et al. 1994).

In order to understand the evolution of the X-ray emission of novae, more systematic observations are required, as exemplified by recent Swift campaigns on RS Oph and V2491 Cyg (Osborne et al. 2009; Bode et al. 2006; K. L. Page et al. 2009, in preparation). Here, we present Swift monitoring observations of the nova V458 Vul, which was discovered by H. Abe 2007 August 8.54 at 9.5 mag (Nakamura et al. 2007; see also Henden \& Munari 2007). Buil \& Fujii (2007) found $\mathrm{P}$ Cygni profiles in $\mathrm{H} \alpha, \beta$, and $\gamma$ lines, as well as in various He I lines with full width at halfmaximum (FWHM) corresponding to $\sim 1700-1900 \mathrm{~km} \mathrm{~s}^{-1}$. On these grounds, they classified it as a classical nova. Images obtained by the IPHAS survey (Drew et al. 2005) shortly before the nova outburst, reveal a point source nova progenitor surrounded by a faint shell. Follow-up imaging and spectroscopy imply this is a slow moving and massive shell of a planetary nebula (PN) as opposed to a fast-moving and much lower mass nova remnant (Wesson et al. 2008). The only other likely example of a classical nova occurring inside a PN was Nova GK Per 1901 (Bode et al. 2004). The optical decline of V458 Vul was observed in detail by Poggiani (2008), and the timescale $t_{2}$ (time for decline by 2 mag) was estimated at 7 days. The maximum magnitude versus rate of decline (MMRD) relationship for the smoothed light curve implies a distance of 6.7-10.3 kpc (Poggiani 2008), and the relation derived by della Valle \& Livio (1995) leads to a distance of $8.8 \pm 0.8 \mathrm{kpc}$. However, the optical light curve showed two strong flarelike peaks on days 4 and 10 after outburst (Tarasova 2007). This complicates the determination of a reliable decline time, and the MMRD relationship method may not work very well for novae with such erratic early light curves. Wesson et al. (2008) found a distance of $13 \mathrm{kpc}$ based on light travel time from the nova to the flash-ionized nebula and other methods, and we adopt this value for our purposes. The interstellar extinction $\left(A_{V, \text { ISM }}\right)$ was estimated to be $1.76 \pm 0.32 \mathrm{mag}$ (Poggiani 2008). This converts to an interstellar hydrogen-equivalent extinction column density of $N_{\mathrm{H}, \mathrm{ISM}}=(3.15 \pm 0.57) \times 10^{21} \mathrm{~cm}^{-2}$ using the relation $N_{\mathrm{H}, \mathrm{ISM}} / A_{V, \mathrm{ISM}}=1.79 \times 10^{21} \mathrm{~cm}^{-2} \mathrm{mag}^{-1}$ (Predehl \& Schmitt 1995). The HEASARC $N_{\mathrm{H}}$ tool $^{20}$ calculates the total Galactic H I column density for any direction using the Leiden/Argentine/Bonn (LAB; Kalberla et al. 2005) and Dickey \& Lockman (1990) Galactic H I surveys. For a cone of radius 0.5 centered on the J(2000) coordinates of V458 Vul, the LAB and Dickey \& Lockman (1990) maps give an average $N_{\mathrm{H}}$ value of

$\overline{20}$ http://heasarc.gsfc.nasa.gov/cgi-bin/Tools/w3nh/w3nh.pl
$3.7 \times 10^{21} \mathrm{~cm}^{-2}$. Lynch et al. (2007) found $E(B-V)=0.6$, which converts to $3.6 \pm 1.2 \times 10^{21} \mathrm{~cm}^{-2}$ using the relation $N_{\mathrm{H}} / E(B-V)=6 \pm 2 \times 10^{21} \mathrm{~cm}^{-2}$ by Dickey \& Lockman (1990) and Bohlin et al. (1978).

A number of photometric periods were reported by Goranskij et al. (2008), and they attributed a periodicity of 0.58946 days ( $14.147 \mathrm{hr}$ ) to the orbital modulation. We caution, however, that different periods have been detected in different observations (Wesson et al. 2008) and finding the orbital period is not a straightforward task. Apart from the two flares in the early optical light curve, Tarasova (2007) found changes in the line widths that were related to these brightenings. Such events have been observed in other novae as well (e.g., in V723 Cas or V2362 Cyg, Goranskij et al. 2007 and Lynch et al. 2008, respectively). The nature of rebrightenings in novae is unclear, and secondary ejection events or interactions with the accretion disk have been proposed (Lynch et al. 2008).

The first X-ray observation of V458 Vul was obtained 1.2 days after outburst ${ }^{21}$ with the X-Ray Telescope (XRT) aboard Swift but yielded no detection (see below). Observations at this early stage of nova evolution are justified by the X-ray detection of V838 Her approximately 5 days after discovery (Lloyd et al. 1992). 70 days later, another Swift observation of V458 Vul was carried out, and Drake et al. (2007) found 192 X-ray counts in a $6.7 \mathrm{ks}$ exposure. From thermal models they estimated a plasma temperature of $\mathrm{k} T=0.2_{-0.07}^{+0.40} \mathrm{keV}$ and an absorbing column of $N_{\mathrm{H}}=8_{-3}^{+2} \times 10^{21} \mathrm{~cm}^{-2}$. Around day 88 after outburst (2007 November 4), a Suzaku observation with medium spectral resolution and higher $\mathrm{S} / \mathrm{N}$ than obtained by $S$ wift was carried out using the X-ray Imaging Spectrometer (XIS; Koyama et al. 2007), and Tsujimoto et al. (2008) found a plasma temperature of $0.64 \pm 0.07 \mathrm{keV}$ and $N_{\mathrm{H}}=3.1_{-1.3}^{+1.8} \times 10^{21} \mathrm{~cm}^{-2}$ from optically thin collision-dominated plasma emission models. Since the Suzaku detectors have higher spectral resolution than the Swift XRT, individual lines can be identified, and the spectrum is clearly an emission line spectrum. Tsujimoto et al. (2008) investigated the chemical composition and found overabundances of $\mathrm{Ne}, \mathrm{Mg}, \mathrm{Si}$, and $\mathrm{S}$, and an underabundance of Fe. For $\mathrm{O}$ they determined an upper limit of 1.5 times solar. On day 397, the nova entered the SSS phase with a highly variable Swift XRT count rate (Drake et al. 2008). The aim of this paper is the study of the evolution of the hard X-ray component that was first observed on day 71 after outburst (Drake et al. 2007). In Section 2, we describe four observing campaigns with optical, ultraviolet, and X-ray observations. For the Swift observations taken after day 397 we only analyze the hard X-ray emission component and postpone the analysis of the SSS phase to a later paper that will be published after the SSS phase has ended. In Section 3, we discuss our results, and summarize our conclusions in Section 4.

\section{OBSERVATIONS}

In this paper, we present 31 Swift XRT (Burrows et al. 2005) and UVOT (Roming et al. 2005) observations taken between 2007 August and 2008 September. The observations are grouped in four campaigns (see Table 1), one observation only 1.2 days after the outburst, 10 observations taken between days 71 and 140.5, eight observations taken between days 315 and 390, and 12 observations taken during the SSS phase, after day

21 All times are given in days after discovery, 2007 August 8.54, and are midtimes between start and end of the observations. 
Table 1

Observation Log (Left) and Results (Right)

\begin{tabular}{|c|c|c|c|c|c|c|c|c|c|c|}
\hline Start Date & Stop Date & Day $^{\mathrm{a}}$ & ObsID & $\begin{array}{l}\text { Net Exp. } \\
\text { (s) }\end{array}$ & $\begin{array}{c}\mathrm{CR}(\text { counts ks} \\
\\
0.25-10 \mathrm{keV}\end{array}$ & $\mathrm{HR}^{\mathrm{c}}$ & $\begin{array}{c}\text { uvw1 }{ }^{\mathrm{d}} \\
2600 \AA^{\mathrm{e}} \\
\pm 693 \AA^{\mathrm{f}}\end{array}$ & $\begin{array}{c}\text { uvw2 } 2^{\mathrm{d}} \\
1928 \AA^{\mathrm{e}} \\
\pm 657 \AA^{\mathrm{f}}\end{array}$ & $\begin{array}{c}\mathrm{uvm} 2^{\mathrm{d}} \\
2246 \AA^{\mathrm{e}} \\
\pm 498 \AA^{\mathrm{f}}\end{array}$ & $\begin{array}{c}u^{\mathrm{d}} \\
3465 \AA^{\mathrm{e}} \\
\pm 785 \AA^{\mathrm{f}}\end{array}$ \\
\hline 2007 Aug 9, 16:27 & Aug 9, 16:43 & 1.19 & 00030980001 & 985.6 & $<3.1$ & $\ldots$ & $\ldots$ & 203.00 & 114.00 & $\ldots$ \\
\hline 2007 Oct $18,01: 06$ & Oct $18,23: 47$ & 71.01 & 00030980002 & 6515.4 & $31.3 \pm 2.5$ & $+0.15 \pm 0.13$ & $\ldots$ & $\ldots$ & $\ldots$ & $\ldots$ \\
\hline 2007 Nov $1,01: 10$ & Nov 1, 02:42 & 84.57 & 00030980003 & 908.1 & $48.8 \pm 8.3$ & $+0.22 \pm 0.30$ & $\ldots$ & 29.40 & $\ldots$ & $\ldots$ \\
\hline 2007 Nov 8, 16:06 & Nov 8, 18:02 & 92.21 & 00030980004 & 2276.7 & $59.0 \pm 5.6$ & $+0.08 \pm 0.15$ & $\ldots$ & $\ldots$ & $\ldots$ & 0.04 \\
\hline 2007 Nov $15,03: 56$ & Nov $15,05: 49$ & 98.70 & 00030980005 & 1967.4 & $67.0 \pm 7.4$ & $+0.11 \pm 0.18$ & 29.00 & $\ldots$ & $\ldots$ & $\ldots$ \\
\hline 2007 Nov 22, 01:13 & Nov 22, 03:07 & 105.58 & 00030980006 & 1710.4 & $52.5 \pm 6.2$ & $-0.21 \pm 0.13$ & $\ldots$ & $\ldots$ & 7.17 & $\ldots$ \\
\hline 2007 Nov 29, 21:11 & Nov 29, 23:12 & 113.42 & 00030980007 & 2861.3 & $57.2 \pm 4.9$ & $-0.1 \pm 0.12$ & $\ldots$ & 23.20 & $\ldots$ & $\ldots$ \\
\hline 2007 Dec 6, 02:46 & Dec 6, 20:23 & 119.98 & 00030980008 & 2237.1 & $62.8 \pm 5.8$ & $-0.1 \pm 0.12$ & $\ldots$ & $\ldots$ & $\ldots$ & 0.06 \\
\hline 2007 Dec $13,19: 28$ & Dec 13, 22:60 & 127.38 & 00030980009 & 1919.7 & $57.4 \pm 6.2$ & $+0.12 \pm 0.17$ & 11.60 & $\ldots$ & $\ldots$ & $\ldots$ \\
\hline 2007 Dec 20, 12:17 & Dec $20,17: 22$ & 134.11 & 00030980010 & 2200.0 & $70.9 \pm 7.1$ & $-0 . \pm 0.14$ & $\ldots$ & $\ldots$ & 6.19 & $\ldots$ \\
\hline 2007 Dec 27, 00:02 & Dec 27, 01:59 & 140.54 & 00030980012 & 2386.7 & $35.9 \pm 5.5$ & $-0.15 \pm 0.18$ & $\ldots$ & 74.30 & $\ldots$ & $\ldots$ \\
\hline 2008 Jun $18,00: 48$ & Jun 18, 23:31 & 315.00 & 00030980013 & 7661.3 & $24.0 \pm 1.9$ & $-0.48 \pm 0.06$ & 2.47 & $\ldots$ & $\ldots$ & $\ldots$ \\
\hline 2008 Jul 15, 21:28 & Jul 15, 23:25 & 342.43 & 00030980014 & 2647.1 & $22.5 \pm 3.4$ & $+0.02 \pm 0.21$ & 3.20 & $\ldots$ & $\ldots$ & $\ldots$ \\
\hline $2008 \mathrm{Jul} 22,14: 20$ & Jul 22, 17:33 & 349.16 & 00030980015 & 1748.6 & $13.0 \pm 3.3$ & $-0.1 \pm 0.34$ & 7.76 & $\ldots$ & $\ldots$ & $\ldots$ \\
\hline 2008 Jul 29, 18:11 & Jul 29, 23:01 & 356.36 & 00030980016 & 3230.7 & $12.7 \pm 2.5$ & $-0.35 \pm 0.17$ & 6.69 & $\ldots$ & $\ldots$ & $\ldots$ \\
\hline 2008 Aug 12, 04:32 & Aug 12, 14:28 & 369.89 & 00030980018 & 4163.0 & $18.5 \pm 2.4$ & $+0.21 \pm 0.22$ & 4.22 & $\ldots$ & $\cdots$ & $\ldots$ \\
\hline 2008 Aug 19, 07:06 & Aug 19, 09:03 & 376.83 & 00030980019 & 2112.7 & $28.4 \pm 4.8$ & $-0.41 \pm 0.10$ & 2.45 & $\ldots$ & $\cdots$ & $\cdots$ \\
\hline 2008 Aug 26, 01:03 & Aug 26, 02:59 & 383.58 & 00030980020 & 1915.4 & $19.1 \pm 3.7$ & $+0.40 \pm 0.38$ & 3.03 & $\ldots$ & $\cdots$ & $\cdots$ \\
\hline 2008 Sep 1, 06:30 & Sep 1, 16:19 & 389.97 & 00030980021 & 4622.5 & $17.7 \pm 2.2$ & $-0.43 \pm 0.10$ & 2.29 & $\cdots$ & $\ldots$ & $\cdots$ \\
\hline 2008 Sep 9, 03:01 & Sep 9, 14:24 & 397.86 & 00030980022 & 3074.2 & $45.2 \pm 4.2$ & $-0.84 \pm 0.02$ & 1.75 & $\ldots$ & $\ldots$ & $\ldots$ \\
\hline 2008 Sep 14, 01:51 & Sep 15, 22:56 & 402.57 & 00030980023 & 6954.9 & $175.0 \pm 5.2$ & $-0.95 \pm 0.00$ & 1.58 & $\ldots$ & $\ldots$ & $\ldots$ \\
\hline 2008 Sep 16, 08:11 & Sep 16, 19:44 & 404.84 & 00030980024 & 4155.5 & $207.3 \pm 7.4$ & $-0.97 \pm 0.00$ & 1.63 & $\ldots$ & $\ldots$ & $\ldots$ \\
\hline 2008 Sep 17, 00:02 & Sep 17, 22:59 & 405.50 & 00030980026 & 12119.3 & $408.2 \pm 5.9$ & $-0.97 \pm 0.00$ & 1.33 & $\cdots$ & $\cdots$ & $\cdots$ \\
\hline 2008 Sep 18, 01:54 & Sep 18, 21:41 & 406.57 & 00030980027 & 10581.0 & $180.8 \pm 4.3$ & $-0.95 \pm 0.00$ & 1.56 & $\ldots$ & $\ldots$ & $\ldots$ \\
\hline 2008, Sep 21, 11:42 & Sep 21, 13:40 & 409.98 & 00030980030 & 2095.2 & $702.3 \pm 18.6$ & $-0.98 \pm 0.00$ & 0.99 & & $\ldots$ & $\ldots$ \\
\hline 2008, Sep 23, 00:51 & Sep 23, 16:49 & 411.53 & 00030980032 & 1860.5 & $631.5 \pm 19.8$ & $-0.99 \pm 0.00$ & 1.15 & & $\ldots$ & $\ldots$ \\
\hline 2008, Sep 25, 12:01 & Sep 25, 17:04 & 413.99 & 00030980033 & 2222.6 & $282.0 \pm 11.5$ & $-0.99 \pm 0.00$ & 1.16 & . & $\ldots$ & $\ldots$ \\
\hline 2008, Sep 30, 03:18 & Sep 30, 19:35 & 418.63 & 00030980034 & 1905.4 & $584.9 \pm 17.8$ & $-0.99 \pm 0.00$ & 1.08 & $\ldots$ & $\ldots$ & $\cdots$ \\
\hline 2008 , Oct $1,06: 10$ & Oct 1, 11:08 & 419.75 & 00030980035 & 2150.2 & $675.8 \pm 18.0$ & $-0.98 \pm 0.00$ & 1.23 & $\cdots$ & $\cdots$ & $\cdots$ \\
\hline 2008 , Oct $2,09: 28$ & Oct $2,16: 13$ & 420.89 & 00030980036 & 2020.3 & $702.6 \pm 18.7$ & $-0.98 \pm 0.00$ & 1.07 & $\cdots$ & $\cdots$ & $\cdots$ \\
\hline 2008 , Oct $3,00: 17$ & Oct $3,03: 42$ & 421.51 & 00030980037 & 1825.5 & $762.6 \pm 22.7$ & $-0.97 \pm 0.00$ & 0.98 & $\cdots$ & $\ldots$ & $\ldots$ \\
\hline
\end{tabular}

Notes.

a After outburst (2007 Aug 8.54), average between start and stop times.

b Corrected for PSF losses and bad columns.

${ }^{\mathrm{c}}$ Hardness ratio $\mathrm{HR}=(H-S) /(H+S)$ with $S=0.25-1 \mathrm{keV}$ and $H=1-10 \mathrm{keV}$.

${ }^{\mathrm{d}}$ Fluxes in $10^{-15} \mathrm{erg} \mathrm{cm}^{-2} \mathrm{~s}^{-1}$.

e Central wavelength (Poole et al. 2008).

${ }^{\mathrm{f}}$ Band width (FWHM; Poole et al. 2008).

390 (Drake et al. 2008). The fourth campaign was a highdensity campaign in response to the emergence of the bright SSS component. In this paper, we analyze only the hard emission component of the observations taken during the fourth campaign and postpone the analysis of the SSS phase because it has not ended at the time of writing. During the third campaign we also obtained dense ground-based optical monitoring observations in the $V$-band.

\subsection{Optical Observations}

Ground-based photometric time series of V458 Vul were obtained during the period July 8 to August 28 using four different $0.28-0.4 \mathrm{~m}$ telescopes located in Wales, England, Belgium, and New Hampshire, US, and are equipped with SBIG or Starlight Express cameras. Images were processed in a standard fashion using MaxIm DL and AIP4WIN, and differential magnitudes were measured using USNO-B1.0 11080459444, 1109-0453942, and 1108-0459876 as comparison stars. Instrumental magnitudes were then converted to a pseudo$V$ band using the AAVSO photometric sequence for V458 Vul.

\subsection{Swift $U V$ and $X$-Ray Observations}

In the left part of Table 1 we list all Swift observations, giving the date and time when each observation started and ended, the corresponding number of days after outburst (averaged between start and stop times), the ObsID, and the exposure time. Due to its low-Earth orbit, Swift can only observe a given target for a maximum of $\sim 2$ ks every 96-minute revolution, and each observation consists of a number of these short snapshots. The XRT observations are integrated over all snapshots, while the UVOT filters can be switched during an observation. The bandpasses for each filter are given in terms of central wavelengths and FWHM in the header of the right part of Table 1.

\subsubsection{Reduction Procedures}

We have used the Swift tools version 2.9 with CALDB 2.9, which are part of the HEADAS software package ${ }^{22}$ to produce level 2 products (sky images for UVOT observations and cleaned event files for XRT observations). UV fluxes were extracted from the sky images using standard tools, and X-ray count

\footnotetext{
22 http://swift.gsfc.nasa.gov/docs/software/lheasoft/
} 


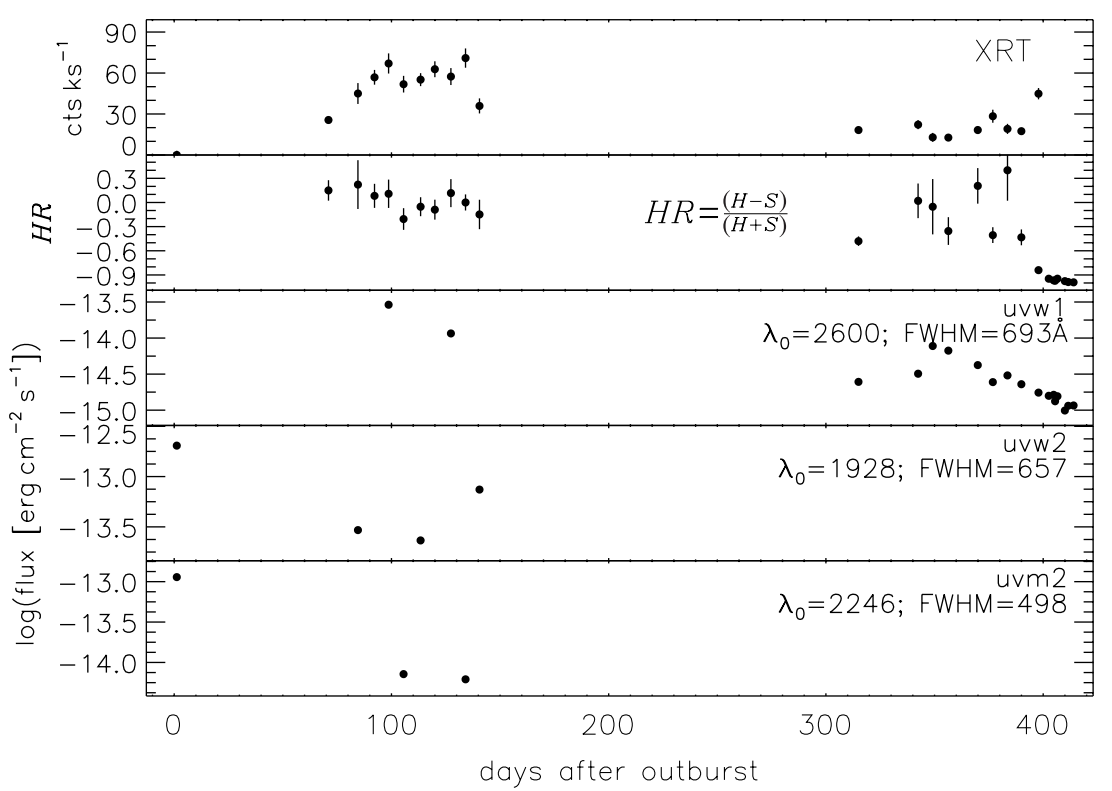

Figure 1. Comparison of the X-ray light curve, X-ray hardness (top two panels; with $S$ and $H$ the count rates in the bands $0.25-1 \mathrm{keV}$ and $1-10 \mathrm{keV}$, respectively), and UV fluxes. After day 400 the X-ray count rate is higher than 90 counts ks ${ }^{-1}$ due to the SSS phase component, and these rates do not fit in the top graph.

rates were obtained from the cleaned event files following the procedure described by Ness et al. (2007).

We have determined the source count rate using a circular source extraction region with radius 10 pixels $\left(=23^{\prime \prime} .6\right)$, and an annular background region with inner and outer radii of 10 and 80 pixels, respectively. This outer radius $\left(=188^{\prime \prime} .8\right)$ was chosen so as to exclude a nearby $\mathrm{X}$-ray source. The analysis procedure adopted from Ness et al. (2007) corrects for source photons contained in the wings of the point-spread function (PSF) that are recorded in the background region. We have also corrected for losses in cases where the source position coincided with bad columns on the detector. The bad columns can be identified on the exposure maps, and we have calculated a correction factor by comparing the effective areas extracted from the level 2 event file and from the exposure map.

In the right part of Table 1, we list the corrected XRT count rates with $1 \sigma$ uncertainties or $95 \%$ upper limits, hardness ratios, and UVOT fluxes. The XRT hardness ratio is defined conventionally here as $\mathrm{HR}=(H-S) /(H+S)$, with $H$ and $S$ being the number of counts in a hard and soft X-ray band, respectively. With this definition, the hardness ratio is +1 for very hard sources and -1 for very soft sources. We have calculated the hardness ratios using an energy band of $0.25-1 \mathrm{keV}$ for $S$ and $1-10 \mathrm{keV}$ for $H$. We chose these ranges to yield a roughly equal number of counts in each band in order to minimize the uncertainties in the hardness ratios.

\subsubsection{Four Observing Campaigns}

In Figure 1, we illustrate the evolution of X-ray count rates and hardness ratios (top two panels) and the UVOT fluxes (in units of $10^{-15} \mathrm{erg} \mathrm{cm}^{-2} \mathrm{~s}^{-1}$, bottom three panels) during the first $\sim 420$ days.

For day 1.2, we find a 95\% upper limit of $0.0021 \mathrm{cps}$. From the spectral models presented in Section 2.3 and Table 2, and assuming a distance of $13 \mathrm{kpc}$, this implies an X-ray luminosity of less than $10^{33} \mathrm{erg} \mathrm{s}^{-1}$ for the XRT band of $0.25-10 \mathrm{keV}$. The uvw2 flux corresponds to a luminosity of $4 \times 10^{33} \mathrm{erg} \mathrm{s}^{-1}$ in the waveband 1271-2585 $\AA$.

From day 71 to day $\sim 100$, the $\mathrm{X}$-ray count rate increases monotonically, while the hardness stays about the same. A small drop in count rate after day $\sim 100$ is accompanied by a reduction in hardness. The spectrum does not evolve into that of an SSS at this time. We have studied the likelihood for the hardness ratio being variable during campaign II. All values are consistent with their mean, and assuming a constant ratio of +0.02 yields a reduced $\chi^{2}=0.8$. The UV flux has significantly decreased from campaign I to II, and the corresponding UV luminosity varies between $5 \times 10^{32} \mathrm{erg} \mathrm{s}^{-1}$ and $1.5 \times 10^{33} \mathrm{erg} \mathrm{s}^{-1}$ in the waveband 1271-2585 $\AA$, thus a factor around 10 lower than in campaign I.

Campaign III starts with a lower X-ray count rate compared to campaign II, but the spectrum is significantly softer. However, 30 days into campaign III, the hardness ratio was back up again, and the observation taken on day 315 is the only campaign III observation that has an SSS component. In campaign III, we have used the same UVOT filter (uvw1) for all observations, and the UV luminosity varies between $6 \times 10^{31} \mathrm{erg} \mathrm{s}^{-1}$ and $1.6 \times 10^{32} \mathrm{erg} \mathrm{s}^{-1}$ in the waveband 1900-3300 $\AA$.

In Figure 2, we present details of the first observation of campaign III (day 315), showing detailed X-ray light curves in two energy bands and the simultaneous uvw1 light curve. Three data points are missing because of tracking problems in orbits 7,8 , and 10 , during which the source was not in the field. We have excluded these orbits for extracting the average count rate listed in Table 1. In the soft band a flarelike event with a peak around day 314.86 and a duration less than 0.4 days is seen which has no counterpart in harder X-rays or the uvw1 fluxes. A similar flare event has been reported by Drake et al. (2003) in the nova V1494 Aql (see also Rohrbach et al. 2009). The hard $\mathrm{X}$-ray count rate seems to be anticorrelated with the uvw1 fluxes, as each increase in X-ray count rate coincides with a decrease in UV brightness. In order to determine whether there was a correlation between the X-ray count rate and the UV fluxes, we have computed a Spearman rank coefficient ${ }^{23}$ of $r_{\mathrm{S}}=-0.43$. This corresponds to a $<2 \sigma$ probability of an anticorrelation which thus cannot be considered statistically significant.

\footnotetext{
${ }^{23}$ Using the Web tool Wessa, P. (2008), Free Statistics Software, Office for Research Development and Education, ver. 1.1.23-r2, URL http://www.wessa.net/
} 


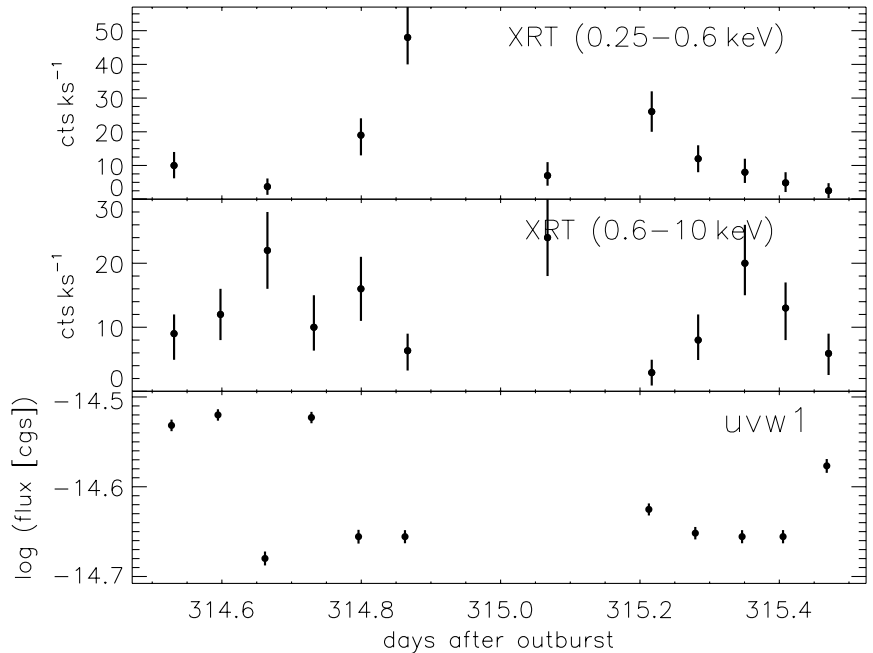

Figure 2. X-ray soft and hard light curves and uvw1 light curve around day 315. A small flare is seen in the soft X-ray component. The hard X-ray count rate and the uvw1 flux are suggestive of an anticorrelation, but a Spearman rank test with 11 values gives a coefficient of -0.43 , which is not significant.

In Figure 3, we show the X-ray and uvw1 data points for all observations taken between days 315 and 390 (campaign III). In order to study only the hard emission, we extracted the $\mathrm{X}$-ray count rates in the bandpass $0.6-10 \mathrm{keV}$. Our choice of energy range here is based on the spectrum that is shown in Figure 6 (dark gray shade), where it can be seen that below $0.6 \mathrm{keV}$ the emission is dominated by the new soft component. For comparison we include the $V$-band magnitudes described in Section 2.1, and they show the same long-term variations as the uvw1 data.

From the top two panels it is again suggestive that the hard $\mathrm{X}$-ray count rates anticorrelate with the uvw1 fluxes. In order to illustrate this anticorrelation, we have rescaled the X-ray count rates to the uvw 1 fluxes and inverted the X-ray light curve. In the bottom panel of Figure 3, we show the inverse X-ray light curve in comparison to the uvw 1 fluxes. The same up and down trends can be seen. The Spearman rank coefficient is $r_{\mathrm{S}}=-0.59$ for all eight data points but $r_{\mathrm{S}}=-0.93$ if excluding the observation taken on day 390. A reason to exclude this data point would be that the UV flux in this observation could already be affected by emission from the WD, as a bright SSS spectrum was observed only a week later (see Figure 4). The latter correlation yields a $97 \%$ probability, which is suggestive of a real anticorrelation.

Next, we have tested the proposed orbital solution given by Goranskij et al. (2008), $\max =2454461.479+0.58946^{\text {days }} \times E$, with max being the barycentric Julian Day of optical maximum. We have converted the arrival times of the photons from all observations taken during campaign III as well as the observing times of the optical $V$-band magnitudes (see Section 2.1), but in none of the bandpasses do we see any evidence for modulation at this period. We performed additional period checks on the uvw1 light curve of days 315-390 but found no convincing evidence for any periodic changes. We note that a period search is difficult with the interrupted light curves available from Swift.

After day 397 the SSS phase started, and a campaign of monitoring at higher cadence was initiated (campaign IV; see Drake et al. 2008). In this paper, we focus on the evolution of the hard component and postpone the discussion of the SSS phase to a future paper. In order to trace the evolution of the hard component separately from the SSS emission, we have extracted the light curves in the energy bands $0.25-0.7 \mathrm{keV}$ and

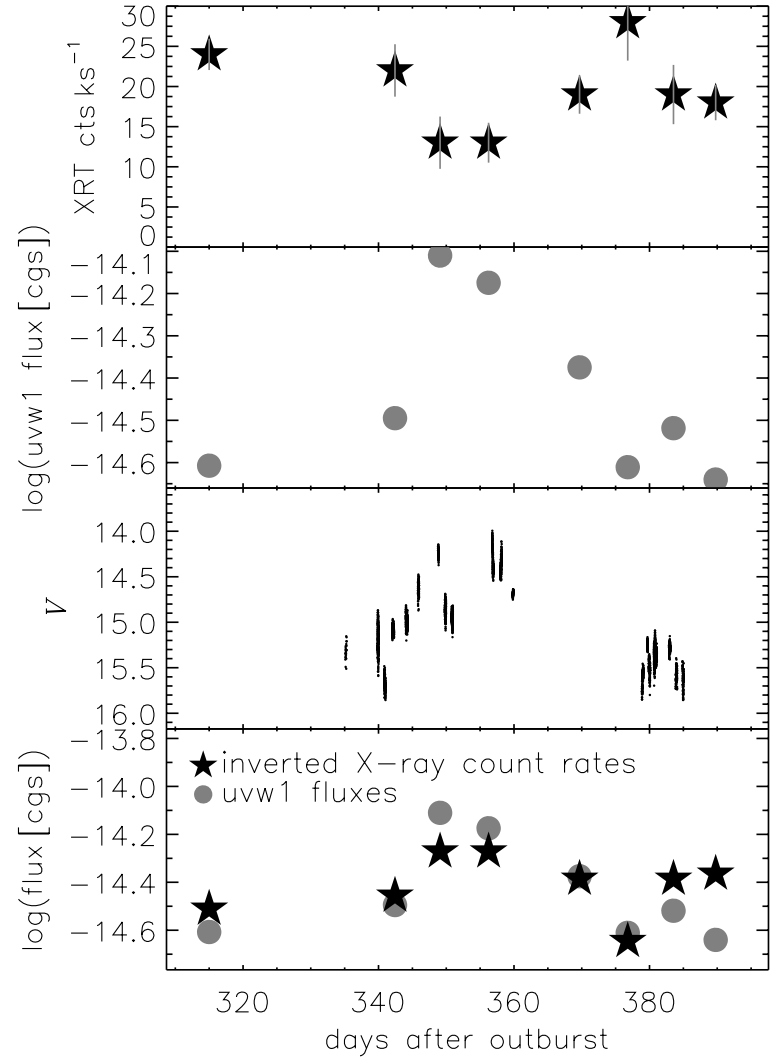

Figure 3. From top to bottom: XRT count rates, uvw1 fluxes, $V$-band magnitudes, and comparison of X-ray and uvw1 fluxes for the observations taken between day 315 and 390 . The X-ray count rates are extracted from the hard band $(0.6-10 \mathrm{keV})$. We have converted the X-ray count rates to scale with the uvw1 fluxes. The comparison of the inverted X-ray light curve with the uvw1 light curve is shown in the bottom panel and illustrates the anticorrelation. The Spearman rank test on the first seven data points yields a $97 \%$ probability that the anticorrelation is real, but with the last data point (day 390) included, the indication for an anticorrelation is much weaker. A reason could be that at that time the UV flux is affected by the emerging SSS component (see the text).

$0.7-10 \mathrm{keV}$, respectively. We chose a higher pivot energy of $0.7 \mathrm{keV}$ in order to exclude any SSS emission from the hard bandpass (see Figure 6). The brightness of the SSS component leads to significant effects of pile up, whereby two incoming $\mathrm{X}$-rays are counted as one count of twice the energy. In order to avoid contamination of the hard component by these counts, we have corrected for pile up by excluding the central 5 pixels for the time intervals when the total count rate was between 0.3 and 0.5 counts $\mathrm{s}^{-1}$, and 7 pixels when it was above 0.5 counts $\mathrm{s}^{-1}$. The extracted count rates have then been upscaled, yielding the PSF-integrated total number of counts. We note, however, that the pile-up correction leads to small raw numbers of counts, which leads to large statistical uncertainties. Detailed analyzes are thus not possible for the faint hard component. In Figure 4, we compare the evolution of the corrected soft $(0.25-0.7 \mathrm{keV})$ and hard (0.7-10 keV) X-ray count rates and of the uvw1 fluxes (top to bottom, respectively). The hard component is still present, and the corrected count rates are consistent with those found in the pre-SSS observations. In the middle panel of Figure 4 one can see that the hard count rate exhibits variability that is uncorrelated to that of the soft flux (top panel). The hard component is thus unaffected by the SSS emission and can be assumed to continue to fade at a very slow rate. However, no significant reduction in count rate can be identified from the middle panel of Figure 4. 


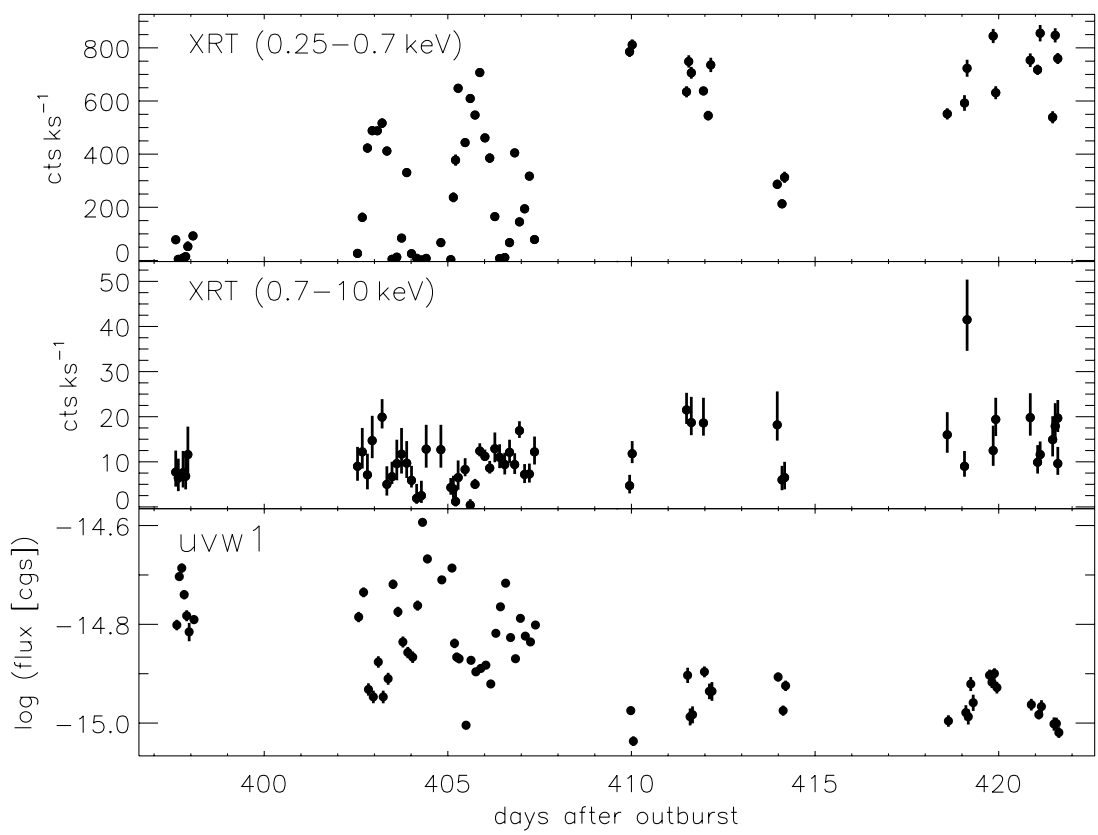

Figure 4. Soft and hard X-ray light curves (top two panels) and the corresponding uvw1 fluxes for campaign IV. The pivot energy is chosen higher to avoid contamination of the hard count rates by SSS emission. All X-ray count rates are corrected for pile up (see the text).

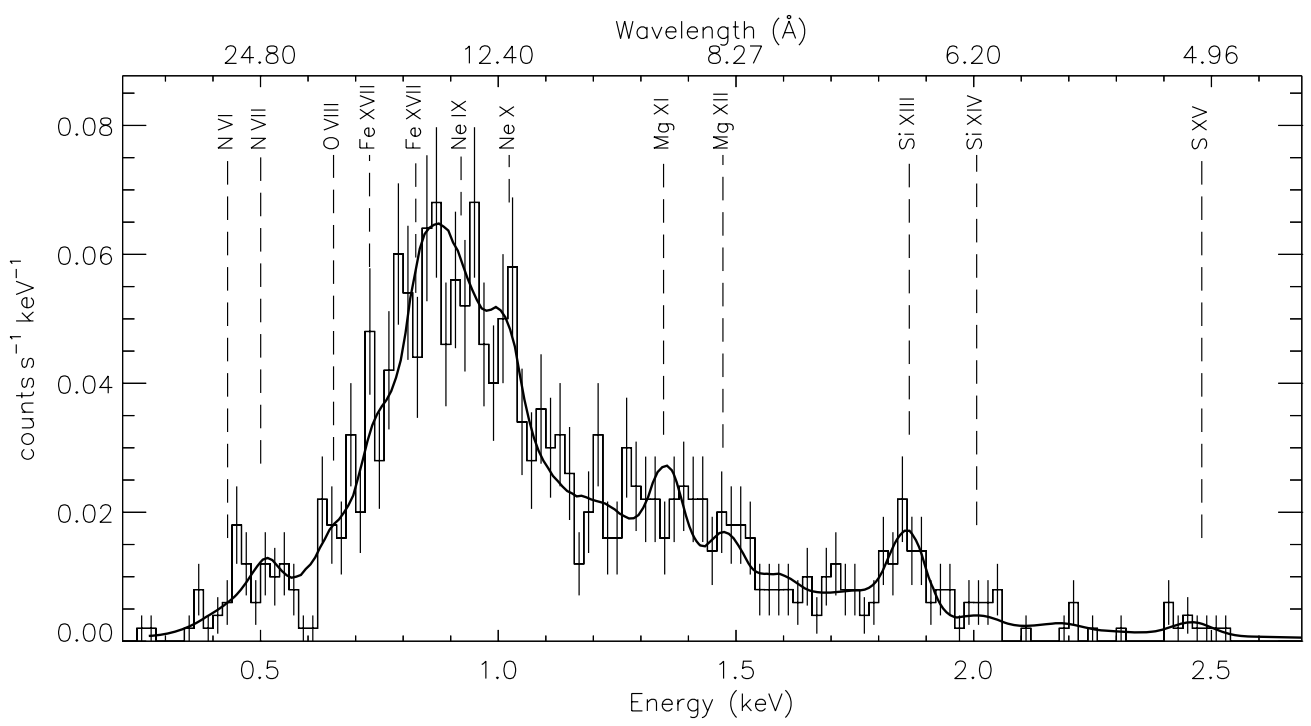

Figure 5. XRT spectrum integrated over days 71-140.5 (total of $25 \mathrm{ks}$ ). The solid line is a thermal model (VAPEC with variable abundances) with the parameters listed in Table 2. Labels are included at energies where strong lines are expected. The instrumental response at $1 \mathrm{keV}$ is $200 \mathrm{eV} \mathrm{FWHM}$, and strong isolated lines can be identified.

\subsection{Spectral Analysis}

In Figure 5, we show the X-ray spectrum integrated over the time interval from day 71 to 140.5 (campaign II). We have included labels at the energies where strong emission lines can in principle arise. Strong emission peaks appear at the wavelengths of silicon and nitrogen lines, while the lines of magnesium are weaker. The FWHM of the XRT spectral response is $\sim 200 \mathrm{eV}$ at $1 \mathrm{keV}$. Between 0.7 and $1.0 \mathrm{keV}$ the H-like and He-like $\mathrm{Ne}$ lines overlap with strong Fe XVII L-shell lines. The combined XRT spectrum resembles the Suzaku spectrum taken on day 88 after outburst (see Tsujimoto et al. 2008).

We have modeled the XRT spectrum using an isothermal VAPEC model (Smith et al. 2001), which represents a collisional, optically thin plasma. We have used the XSPEC fitting package, version 11.3.2ag (Arnaud 1996), allowing variable abundances for $\mathrm{N}, \mathrm{O}, \mathrm{Ne}, \mathrm{Mg}, \mathrm{Si}, \mathrm{S}$, and $\mathrm{Fe}$, with the abundances of all other elements fixed at the solar values of Anders \& Grevesse (1989). In order to account for absorption in the interstellar medium (ISM) we have used the TBABS module with the single parameter $N_{\mathrm{H}}$, the neutral hydrogen column density in $\mathrm{cm}^{-2}$, assuming solar composition of the ISM (Wilms et al. 2000). We have optimized the parameters using CSTAT statistics with which we avoid having to rebin the spectrum. We have used the ERROR command to compute $90 \%$ uncertainty ranges.

The results and $90 \%$ uncertainty ranges for each parameter are listed in Table 2. The temperature, $\mathrm{k} T$, neutral hydrogen column density, $N_{\mathrm{H}}$, element abundances, and X-ray luminosity are in good agreement with the parameters found from Suzaku spectra by Tsujimoto et al. (2008), indicating little evolution 
Table 2

Spectral Models to XRT Spectra of Days 71-140.5 and 315

\begin{tabular}{|c|c|c|}
\hline Parameter & Unit & Value $^{\mathrm{a}}$ \\
\hline \multicolumn{3}{|c|}{ VAPEC model to campaign II (days 71-140.4), combined XRT spectrum } \\
\hline $\mathrm{k} T$ & $\mathrm{keV}$ & $0.64 \pm 0.06$ \\
\hline$N_{\mathrm{H}}$ & $10^{21} \mathrm{~cm}^{-2}$ & $2.7 \pm 0.9$ \\
\hline $\mathrm{A}(\mathrm{N})$ & solar $^{\mathrm{b}}$ & $<27.9$ \\
\hline $\mathrm{A}(\mathrm{O})$ & solar ${ }^{\mathrm{b}}$ & $<1.1$ \\
\hline $\mathrm{A}(\mathrm{Ne})$ & solar $^{\mathrm{b}}$ & $<0.9$ \\
\hline $\mathrm{A}(\mathrm{Mg})$ & solar $^{\mathrm{b}}$ & $0.5 \pm 0.3$ \\
\hline $\mathrm{A}(\mathrm{Si})$ & solar $^{\mathrm{b}}$ & $0.8_{-0.4}^{+1.0}$ \\
\hline $\mathrm{A}(\mathrm{S})$ & solar $^{\mathrm{b}}$ & $0.7_{-0.5}^{+1.0}$ \\
\hline $\mathrm{A}(\mathrm{Fe})$ & solar $^{\mathrm{b}}$ & $0.2_{-0.1}^{+0.3}$ \\
\hline Flux $^{\mathrm{c}}$ & $10^{-12} \mathrm{erg} \mathrm{cm}^{-2} \mathrm{~s}^{-1}$ & $1.15 \pm 0.65$ \\
\hline$L_{X}^{\mathrm{c}, \mathrm{d}}$ & $10^{34} \mathrm{erg} \mathrm{s}^{-1}$ & $2.3 \pm 1.3$ \\
\hline$\chi_{\text {red }}^{\frac{A}{2}}($ dof $)$ & & $0.85(263)$ \\
\hline \multicolumn{3}{|c|}{ Blackbody (bb) plus VAPEC fit to day 315 spectrum } \\
\hline$N_{\mathrm{H}}$ & $10^{21} \mathrm{~cm}^{-2}$ & $3.1 \pm 0.4$ \\
\hline$T_{\mathrm{eff}}(\mathrm{bb})$ & $\mathrm{eV}$ & $22.8_{-5.1}^{+9.4}$ \\
\hline $\log \left(L_{\text {bol }}\right)^{\mathrm{d}}$ & $\operatorname{erg~s}^{-1}$ & $39 \pm 2$ \\
\hline Radius $^{\mathrm{d}}$ & $10^{3} \mathrm{~km}$ & $170_{-161}^{+2640}$ \\
\hline $\mathrm{k} T(\mathrm{VAPEC})$ & $\mathrm{eV}$ & 0.55 \\
\hline Flux $^{\mathrm{c}}(\mathrm{VAPEC})$ & $10^{-12} \mathrm{erg} \mathrm{cm}^{-2} \mathrm{~s}^{-1}$ & $0.30 \pm 0.15$ \\
\hline
\end{tabular}

Notes.

a $90 \%$ uncertainty ranges.

b Anders \& Grevesse (1989).

${ }^{c}$ Unabsorbed over range $0.25-10 \mathrm{keV}$.

d Assuming distance $13 \mathrm{kpc}$.

in the hot plasma between the respective epochs of the Swift average spectrum analyzed here (days 71-141) and the Suzaku (day 88) spectra. From the average count rate we determine a rough count rate-to-luminosity conversion factor of $5 \times 10^{35}$ $\mathrm{erg} \mathrm{s}^{-1} \mathrm{cps}^{-1}$, thus the upper limit of 0.002 counts $\mathrm{s}^{-1}$ for day 1.2 converts to an upper limit to the luminosity of $10^{33} \mathrm{erg} \mathrm{s}^{-1}$ over $0.25-10 \mathrm{keV}$. Element abundances are all consistent with solar values, except for that of $\mathrm{Fe}$, which we find to be at least a factor of 2 lower. Underabundance of $\mathrm{Fe}$ is possibly common in post-asymptotic giant branch (post-AGB) stars, which have undergone dredge-up of s-processed material. Low Fe abundances have also been found in the X-ray spectra of novae, e.g., RS Oph (Ness et al. 2009) and V382 Vel (Ness et al. 2005). Unfortunately, abundances of $\mathrm{N}$ and $\mathrm{O}$ could not be usefully constrained. The upper limit found for $\mathrm{N}$ is relatively high, and allows for the possibility that the hot gas is enriched by $\mathrm{CNO}$ processing. The plasma temperature lies in the range $0.58-0.7 \mathrm{keV}$.

The best-fit model is included with a solid line in Figure 5. The model represents a satisfactory fit. To give a goodness criterion, we have calculated a value of reduced $\chi^{2}=0.85$ (263 degrees of freedom), using the errors extracted with the spectrum in the original binning. In addition, we have carried out the GOODNESS command with 10,000 trials, and found that $41 \%$ of realizations have a fit statistic better than the best fit. For comparison, the "ideal" fit yields $50 \%$ of all realizations in the Monte Carlo calculation to be better, i.e., our fit is formally acceptable.

In Figure 6, we compare the sum of all campaign II XRT spectra (solid line, see Figure 5), the observation taken on the first day of campaign III (day 315 in dark shading), and the sum of the last seven campaign III observations (days 342.4-390 in light shading). From day 140.5 to 315 , the hard component between 0.7 and $1.0 \mathrm{keV}$ has faded, but below $0.5 \mathrm{keV}$ a significantly higher emission level was observed on day 315 . This soft component leads to the lower hardness ratio listed in
Table 1. After day 342.4 the hardness ratio is higher again, and the soft component is not present in the combined XRT spectrum from days 342.4 to 390 . However, the hard component has not changed from day 315 to the summed observation of days 342.4390.

We have fitted this soft component with a blackbody. In order to constrain the blackbody fit against arbitrarily high temperatures, we have added a VAPEC model with the abundances fixed at the same values as those listed in Table 2 . The bestfit blackbody plus VAPEC model is shown with a thin solid line in Figure 6, and the results are given in the lower part of Table 2. The blackbody temperature is reminiscent of a typical SSS spectrum and the value of $N_{\mathrm{H}}$ is consistent with that found from campaign II. The luminosity range encompasses the Eddington luminosity of a $1 M_{\odot}$ white dwarf, but it is not well constrained owing to the uncertainty in effective temperature and $N_{\mathrm{H}}$. Similarly, the radius derived from the temperature and luminosity is very uncertain and is consistent with an extended WD.

In the spectrum taken on day 315 , the hard component above $0.6 \mathrm{keV}$ is difficult to model owing to lack of signal. We find a temperature of the VAPEC component that is consistent with that found from the sum of the campaign-II observations (top part of Table 2). The flux of the VAPEC component is a factor 3.8 lower than that seen in the previous observation on day 315 , and the hard component has thus faded by this amount. The light gray-shaded spectrum shown in Figure 6 indicates the sum of the XRT spectra over days 342.4-390, and there is no significant difference between the dark and light-shaded spectra except for the disappearance of the soft component. This indicates that the hard component evolves slowly.

\section{DISCUSSION}

The classical nova V458 Vul has been monitored in Xrays and the ultraviolet between days 1 and 422 after outburst using the Swift satellite. No X-ray emission was detected 1.2 days after outburst, but the source was bright in the UV. The early optical light curve shows two strong peaks on days 4 and 10 after outburst with dramatic changes in linewidth velocities (Tarasova 2007). Since no X-ray emission was detected three days before the first peak, it is possible that the peaks were in some way associated with the trigger for the $\mathrm{X}$-ray emission. However, no X-ray observations were taken during and immediately after the peaks occurred, and we cannot discuss whether this peculiar behavior is in any way related to the slowly evolving hard X-ray emission. We thus encourage systematic monitoring through this evolutionary phase of future nova outbursts in order that such scenarios can be confirmed or rejected.

The second monitoring campaign took place between days 71 and 140.5 after outburst, and a variable, hard X-ray source was observed. We have modeled the combined X-ray spectrum and found a good fit with an isothermal collision-dominated optically thin plasma model. Our model parameters are consistent with the spectral model fits to Suzaku spectra obtained on day 88 after outburst (see Tsujimoto et al. 2008). We find a chemical composition consistent with a solar mixture, except for $\mathrm{Fe}$, which appears underabundant by a factor of at least 2 . In CNO-cycled material, nitrogen is expected to be overabundant, however, our data allow us only to derive an upper limit to the $\mathrm{N}$ abundance. This limit is high enough that $\mathrm{CNO}$ ashes are not ruled out from the observations. The low Fe abundance might reflect the composition of the post-AGB progenitor star of the 


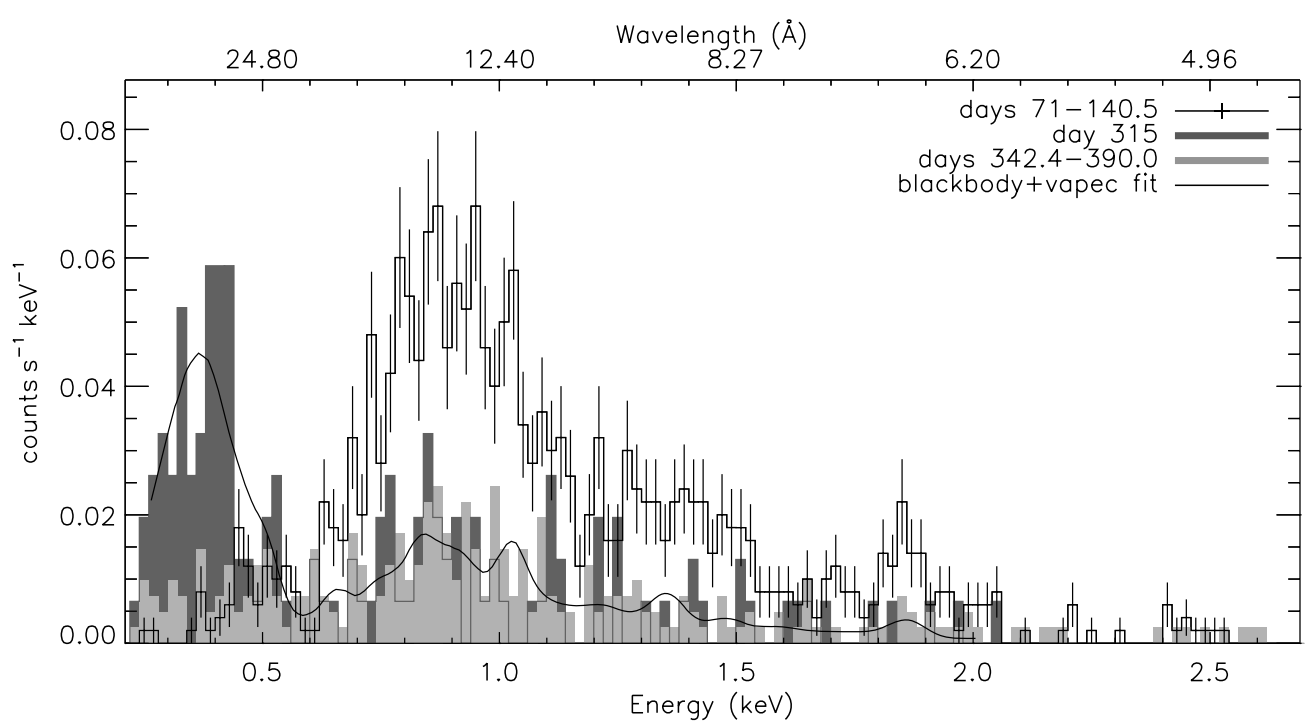

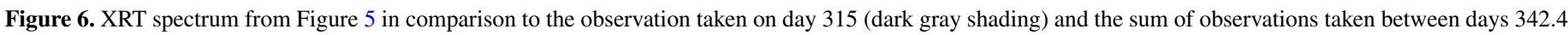
and 390 (light gray shading). Overplotted is a model consisting of a blackbody and a VAPEC with parameters given in Table 2.

WD, and Fe underabundance has been found in several other novae as well.

The first observation of the third campaign between 315 and 390 days after outburst shows the first glimpse of the SSS phase, but the proper SSS phase does not start until 80 days later (Drake et al. 2008). The soft component (0.25$0.6 \mathrm{keV}$ ) is highly variable with a flarelike event in X-rays, while the hard component $(0.6-10 \mathrm{keV})$ shows a different pattern of variability.

We have searched for the orbital period proposed by Goranskij et al. (2008) but found no evidence for it in either X-ray, UV, or optical. Variations seen in the UV are in closer relationship to those in the harder X-ray component than in the soft band. During the entire third campaign, the UV brightness is anticorrelated with the hard X-ray count rate at the $97 \%$ probability level. The hard component during campaign III is a factor 3.8 fainter than during campaign II and can also be modeled with an optically thin plasma spectrum. The $\mathrm{S} / \mathrm{N}$ does not allow us to detect any changes in temperature. If the emission originates from the same plasma as in campaign II, then it has only cooled slightly, and the hard component fades extremely slowly. Owing to additional short-term variability within each observing campaign (see Figure 1), we cannot determine an analytic decay law.

The fourth campaign is dominated by the SSS emission (Drake et al. 2008). At the time of writing, the SSS phase has not ended yet, and we postpone the analysis of the entire SSS phase. Meanwhile, the hard component appears virtually unaffected, which is consistent with expectations that it originates in regions far away from the photosphere of the WD. Our finding of anticorrelations between the hard component and the UV flux suggests that the UV emission originates at least partly from those outer regions as well. However, the fact that the UV emission also correlates with the SSS emission (Drake et al. 2008) suggests that we are seeing a mixture of different UV sources. This may explain why the detection of the anticorrelation between X-ray and UV emission during campaign III is so difficult.

While the SSS phase is the most prominent evolutionary phase in X-rays, additional X-ray production mechanisms are poorly understood. While we are not able to derive a decay law, it is clear that the hard X-ray component fades over a timescale of more than a year and is still present after the SSS phase started. Since the density in the ejecta must have decreased for the SSS spectrum to emerge, X-ray emission originating from shocks within the ejecta might be expected to have faded by this time (Lloyd et al. 1992; O'Brien et al. 1994), yielding a shorter timescale than that observed. However, it is possible that the emission observed in campaign II could have its origin in internal shocks as discussed by Tsujimoto et al. (2008). In that case the hard component observed during campaigns III and IV would have a different origin. On the other hand, Orio et al. (1996) state that they cannot be definitive either about their late-time single epoch detection of Pup 1991 coming from internal shocks in the ejecta or from accretion at a high rate.

Other sources of the hard X-ray emission could have been shock interactions of the expanding ejecta with the stellar wind of the companion or with the surrounding medium which includes the innermost regions of the planetary nebula as is suggested for GK Per from direct imaging (see Bode et al. 2004 and references therein; and also Balman 2005 and Vrielmann et al. 2005). Shocks with the stellar wind of the companion occur most notably in the case of long-period symbiotic novae in which the mass-donor star is an evolved object with a massive wind (e.g., RS Oph; Bode et al. 2006). However, Wesson et al. (2008) note that the pre-outburst colors are consistent with an O-type spectral class, and attribute this to either the post-AGB evolution of the PN progenitor, or to an accretion disk. It is possible that the more tenuous circumstellar environment of this system still provides for significant shock-induced X-rays. The timescale for dissipation of the explosion energy in such a tenuous medium would also be much longer than in the denser wind of the symbiotic case.

\section{SUMMARY AND CONCLUSIONS}

The four observing campaigns can be summarized as follows.

1. The early evolution, before day 70 after outburst, showed an erratic optical light curve (Tarasova 2007), but we have only one X-ray observation on day 1.2 that yields a nondetection. This nondetection suggests that the hard X-ray component 
has not started its evolution with the outburst directly. The two peaks in the optical light curve on days 4 and 10 after outburst could be related to the trigger for the X-ray emission. For example, X-ray and optical emission could have been produced on impact of the expanding ejecta with denser regions of the ambient medium, possibly related to the proposed planetary nebula. However, in order to test such scenarios, denser coverage in X-rays during the early evolution is needed.

2. The first X-ray detection on day 71 after outburst is not an SSS spectrum, but a hard component that continues to increase until $\sim$ day 100 after outburst. The X-ray spectrum is consistent with collisional plasma, yielding typical nova abundances. The X-ray light curve is variable during the second campaign from days 71 to 140.5 .

3. The third campaign, starting on day 315 after outburst, shows a first appearance of a SSS component, but this component disappears again, and is not observed again until day 397 after outburst. We see a suggestion of an anticorrelation between the hard component and the UV emission, which would indicate that the UV emission may at least partially originate from the same outer regions as the hard X-ray emission.

4. The fourth campaign contains a bright SSS component that is not analyzed in this paper. The hard X-ray component is still present, suggesting that it is unaffected by the SSS component, and that it fades extremely slowly. If the UV flux is anticorrelated to the SSS component and to the hard X-ray component (see above) then the observed UV flux originates from the photosphere around the WD and from the outer regions at the same time.

We have discussed several production mechanisms for the hard X-ray component. For internal shocks within the ejecta we expect a shorter decay time than observed (Lloyd et al. 1992; O'Brien et al. 1994), however, it is possible that the earlier hard $\mathrm{X}$-ray emission (before day 140) could have originated from within the ejecta (Tsujimoto et al. 2009). Detectable emission from shocks arising from ejecta interaction with a stellar wind would require a red giant secondary as in symbiotic novae. If this nova occurred within a PN as suggested by Wesson et al. (2008), then the interaction of the nova ejecta with the innermost regions of material associated with the ejection of the PN may be the most plausible source of the long-lasting hard X-ray emission.

We thank the Swift PI Neil Gehrels and Swift schedulers for their support of these TOO observations. J.-U.N. and M.T. gratefully acknowledge support provided by NASA through Chandra Postdoctoral Fellowship grants PF5-60039 and PF6-70044, respectively, awarded by the Chandra X-ray Center, which is operated by the Smithsonian Astrophysical Observatory for NASA under contract NAS8-03060. J.J.D. was supported by NASA contract NAS8-39073 to the Chandra X-ray Center during the course of this research. D.T. acknowledges support from a JSPS fellowship. S.S. received partial support from NSF and NASA grants to ASU. K.L.P., A.P.B, P.A.E., and J.P.O. acknowledge support from STFC. D.S. acknowledges an STFC Advanced Fellowship.

\section{REFERENCES}

Anders, E., \& Grevesse, N. 1989, Geochim. Cosmochim. Acta, 53, 197

Arnaud, K. A. 1996, in ASP Conf. Ser. 101, Astronomical Data Analysis Software and Systems V, ed. G. H. Jacoby \& J. Barnes (San Francisco, CA: ASP), 17

Balman, S. 2005, ApJ, 627, 933

Balman, Ş., Krautter, J., \& Ögelman, H. 1998, ApJ, 499, 395

Bode, M. F., O’Brien, T. J., \& Simpson, M. 2004, ApJ, 600, L63

Bode, M. F., et al. 2006, ApJ, 652, 629

Bohlin, R. C., Savage, B. D., \& Drake, J. F. 1978, ApJ, 224, 132

Buil, C., \& Fujii, M. 2007, Cent. Bur. Electron. Tel., 8862, 2

Burrows, D. N., et al. 2005, Space Sci. Rev., 120, 165

della Valle, M., \& Livio, M. 1995, ApJ, 452, 704

Dickey, J. M., \& Lockman, F. J. 1990, ARA\&A, 28, 215

Drake, J. J., et al. 2003, ApJ, 584, 448

Drake, J. J., et al. 2007, Astron. Tel., 1246, 1

Drake, J. J., et al. 2008, Astron. Tel., 1721, 1

Drew, J. E., et al. 2005, MNRAS, 362, 753

Gallagher, J. S., \& Starrfield, S. 1978, ARA\&A, 16, 171

Goranskij, V. P., Metlova, N. V., Baruskova, E. A., Burenskov, A. N., \& Soloviev, V. Y. 2008, Astron. Tel., 1631, 1

Goranskij, V. P., et al. 2007, Astrophys. Bull., 62, 125

Henden, A., \& Munari, U. 2007, Inf. Bull. Var. Stars, 5803, 1

Kahabka, P., \& van den Heuvel, E. P. J. 1997, ARA\&A, 35, 69

Kalberla, P. M. W., Burton, W. B., Hartmann, D., Arnal, E. M., Bajaja, E., Morras, R., \& Pöppel, W. G. L. 2005, A\&A, 440, 775

Koyama, K., et al. 2007, PASJ, 59, 23

Krautter, J., Ögelman, H., Starrfield, S., Wichmann, R., \& Pfeffermann, E. 1996, ApJ, 456, 788

Lloyd, H. M., O’Brien, T. J., Bode, M. F., Predehl, P., Schmitt, J. H. M. M., Truemper, J., Watson, M. G., \& Pounds, K. A. 1992, Nature, 356, 222

Lynch, D. K., Russell, R. W., Rudy, R. J., \& Woodward, C. E. 2007, IAU Circ., 8883,1

Lynch, D. K., et al. 2008, AJ, 136, 1815

Mukai, K., \& Ishida, M. 2001, ApJ, 551, 1024

Nakamura, Y., Yamaoka, H., Dillon, W. G., Guido, E., Sostero, G., Abe, H., Nakano, S., \& Labordena, C. 2007, Cent. Bur. Electron. Tel., 1029, 2

Ness, J.-U., Schwarz, G. J., Retter, A., Starrfield, S., Schmitt, J. H. M. M., Gehrels, N., Burrows, D., \& Osborne, J. P. 2007, ApJ, 663, 505

Ness, J.-U., Starrfield, S., Jordan, C., Krautter, J., \& Schmitt, J. H. M. M. 2005, MNRAS, 364, 1015

Ness, J.-U., et al. 2009, AJ, 137, 3414

O’Brien, T. J., Lloyd, H. M., \& Bode, M. F. 1994, MNRAS, 271, 155

Orio, M., Balman, S., della Valle, M., Gallagher, J., \& Oegelman, H. 1996, ApJ, 466,410

Orio, M., et al. 2001, MNRAS, 326, L13

Osborne, J. P., et al. 2009, ApJ, submitted

Poggiani, R. 2008, Ap\&SS, 315, 79

Poole, T. S., et al. 2008, MNRAS, 383, 627

Predehl, P., \& Schmitt, J. H. M. M. 1995, A\&A, 293, 889

Rohrbach, J., Ness, J. U., \& Starrfield, S. 2009, AJ, in press

Roming, P. W. A., et al. 2005, Space Sci. Rev., 120, 95

Smith, R. K., Brickhouse, N. S., Liedahl, D. A., \& Raymond, J. C. 2001, ApJ, 556, L91

Starrfield, S., Iliadis, C., \& Hix, W. R. 2008, in Classical Novae, ed. M. Bode \& A. Evans (Cambridge: Cambridge Univ. Press), 77

Tarasova, T. N. 2007, Inf. Bull. Var. Stars, 5807, 1

Tsujimoto, M., Takei, D., Drake, J. J., Ness, J.-U., \& Kitamoto, S. 2009, PASJ, 61,69

Vrielmann, S., Ness, J.-U., \& Schmitt, J. H. M. M. 2005, A\&A, 439, 287

Wesson, R., et al. 2008, ApJ, 688, L21

Wilms, J., Allen, A., \& McCray, R. 2000, ApJ, 542, 914 